\title{
Expression of thyroid transcription factor-1 in normal endometrium is associated with risk of endometrial cancer development
}

\author{
Peggy S Sullivan ${ }^{1}$, Erin L Maresh ${ }^{1}$, David B Seligson ${ }^{1}$, Omar Habeeb ${ }^{1}$, Madhuri Wadehra ${ }^{1}$, \\ Lee Goodglick ${ }^{1}$ and Oliver Dorigo ${ }^{2}$ \\ ${ }^{1}$ Department of Pathology and Laboratory Medicine, UCLA David Geffen School of Medicine, \\ Los Angeles, CA, USA and ${ }^{2}$ Division Gynecologic Oncology, Department of Obstetrics and Gynecology, \\ UCLA David Geffen School of Medicine, Los Angeles, CA, USA
}

\begin{abstract}
Thyroid transcription factor-1 (TTF-1) is a DNA-binding protein that is mainly expressed in thyroid and lung tissue, but has also been found in gynecologic tissue. Recent studies have suggested that TTF-1 has tumor suppressor function in lung adenocarcinoma models. In the current study, we examined whether expression of TTF-1 in benign endometrium and endometrial hyperplasia might impact on the risk of developing endometrial cancer. Formalin-fixed paraffin-embedded endometrial tissues obtained from 535 cases were used to construct an endometrial tissue microarray. One hundred fifty of 207 patients had multiple serial endometrial specimens including 46 patients who progressed to endometrial cancer. The tissue microarray included a range of histopathologies including benign endometrium $(n=231)$, simple hyperplasia $(n=105)$, complex hyperplasia ( $n=36)$, simple atypical hyperplasia $(n=10)$, complex atypical hyperplasia $(n=44)$, and endometrial carcinoma $(n=109)$. Expression of TTF-1 by immunohistochemistry in benign endometrium and endometrial hyperplasia was correlated with progression to cancer and clinical features known to be associated with increased risk of developing endometrial cancer. Carcinoma specimens showed a significantly greater expression of TTF-1 compared with benign endometrium and non-atypical hyperplasia $(P=0.0007$ and $P=0.05)$. Presence of TTF-1 expression in benign endometrium was associated with a significantly decreased risk of cancer development $(P=0.003$, hazards ratio $=0.104,95 \% \mathrm{Cl}: 0.024-0.455)$. TTF-1 expression in hyperplasia did not significantly correlate with progression to cancer. The data from our study show that TTF-1 expression in normal endometrium is associated with a reduced risk of endometrial cancer development. This observation suggests that TTF-1 might function as a tumor suppressor in endometrial tissue. TTF-1 expression in normal endometrium could potentially provide clinically useful information as a biomarker for the risk of endometrial cancer. Modern Pathology (2012) 25, 1140-1148; doi:10.1038/modpathol.2012.64; published online 30 March 2012
\end{abstract}

Keywords: endometrial cancer; endometrial hyperplasia; immunohistochemistry; normal endometrium; TTF-1

Endometrial carcinoma is the most common gynecologic malignancy in the United States with an estimated incidence of 46470 new cases in $2011 .^{1}$ Predisposing risk factors include obesity, nulliparity, endogenous or exogenous estrogen excess, diabetes mellitus, hypertension, early menarche,

Correspondence: Dr PS Sullivan, MD, Department of Pathology and Laboratory Medicine, UCLA David Geffen School of Medicine, 10833 Le Conte Avenue, CHS A7-149, Los Angeles, CA 90095-1732, USA.

E-mail: psullivan@mednet.ucla.edu

Received 8 January 2012; revised 17 February 2012; accepted 19 February 2012; published online 30 March 2012 and late menopause, while smoking and the use of oral contraceptives decrease the risk. ${ }^{2}$

Prior to the development of carcinoma, biopsies may reveal benign endometrium or hyperplastic lesions with or without atypia. The presence of atypical endometrial cells and a complex histopathologic architecture increases the risk of progressing to cancer, but there is generally poor reproducibility in pathologic assessment of endometrial lesions. ${ }^{3-5}$ Biomarkers to predict the progression of a benign or hyperplastic endometrial lesion to cancer have not been identified yet, but would provide an important diagnostic aid for the prevention of endometrial cancer development. 
Thyroid transcription factor-1 (TTF-1) is a DNAbinding protein that controls the expression of thyroid, lung, and rostral brain-specific genes, and plays a critical role in embryogenesis of these organs. It is the product of the gene NK2 homeobox 1 with the gene symbol NKX2-1 on 14q13.3, and it is essential for the expression of several proteins including thyroglobulin, thyroperoxidase, surfactant protein A, and Clara cell secretory protein. ${ }^{6}$ TTF-1 is expressed in both normal and neoplastic lung and thyroid tissues and is often considered a reliable marker of origination from either of these sites. ${ }^{7}$ TTF-1 has also been found to be expressed in small cell carcinomas of the prostate, bladder, cervix, and breast. ${ }^{8,9}$ Recently, TTF-1 expression has been reported in various carcinomas of gynecologic origin including uterine cervix, endometrium, and ovary. ${ }^{10-15}$ The incidence of TTF-1 expression in endometrial malignancies has been reported to range between 0 and $82 \%,{ }^{10-15}$ but the association of TTF-1 with survival has not been studied.

Despite a number of studies that have described the expression of TTF-1 in cancer disease, the biological role of TTF-1 is still unclear. Interestingly, recent studies have suggested that TTF-1 might have tumor suppressor function in lung adenocarcinoma models. ${ }^{16,17}$ Winslow et $a l^{17}$ demonstrated in a murine lung adenocarcinoma model that TTF-1 (also called Nkx2-1) expression decreased the metastatic potential of tumors in vivo. Based on these observations, we hypothesized that TTF-1 might mediate similar effects in normal and hyperplastic endometrium and potentially decrease the risk of progression to endometrial cancer. Using an endometrial cancer progression array, we assessed whether the expression of TTF-1 in benign and endometrial cancer precursor lesions modulates the risk of developing endometrial cancer.

\section{Materials and methods}

\section{Tissue Microarray}

We used an early endometrioid carcinoma progression tissue microarray that was described previously. ${ }^{18}$ Briefly, 535 formalin-fixed, paraffinembedded archival endometrial tissue samples were randomly collected from 207 patients by curettage, biopsy, or hysterectomy procedures dating from 1982 to 2002 (Department of Pathology and Laboratory Medicine, UCLA Medical Center) with institutional review board approval. Of the 207 patients, 150 had at least two specimens collected during separate procedures, for a total of 457 'serial' specimens. Of the 150 patients with multiple serial endometrial specimens in the tissue microarray, 46 patients had progression to endometrial cancer with at least 1 year of follow-up. Three $1.0 \mathrm{~mm}$ diameter cores were sampled from each case for tissue microarray construction, as available, and primary histology was verified by a gynecologic pathologist (PSS). The primary histology included benign endometrium $(n=231)$, simple hyperplasia $(n=105)$, complex hyperplasia $(n=36)$, simple atypical hyperplasia $(n=10)$, complex atypical hyperplasia $(n=44)$, and primary endometrial carcinoma $(n=109)$.

All cases of hyperplasia, atypical hyperplasia, and carcinoma were classified using criteria from the World Health Organization. ${ }^{19}$ All endometrial carcinoma cases were adenocarcinomas of endometrioid type.

\section{Immunohistochemistry}

Paraffin-embedded formalin-fixed sections were baked at $60{ }^{\circ} \mathrm{C}$ for $30 \mathrm{~min}$. The slides were deparaffinized with xylene and rehydrated through graded ethanol. Endogenous peroxidase activity was blocked with $3 \%$ hydrogen peroxide in methanol for $10 \mathrm{~min}$. Heat-induced antigen retrieval was carried out for all sections in 0.001 M EDTA buffer, $\mathrm{pH}=8.0$. Mouse monoclonal antibody for TTF-1 (8G7G3/1, Dako, Carpinteria, CA, USA) was applied at 1:300 titer for $45 \mathrm{~min}$. Slides were subsequently incubated for $30 \mathrm{~min}$ with labeled polymer HRP goat anti-mouse (Dakocytomation Envision System Labeled Polymer HRP). The sections were treated with $\mathrm{DAB}$ for $10 \mathrm{~min}$ for visualization and then counterstained with hematoxylin. Sections were dehydrated through graded ethanol and cover slipped. As TTF-1 is a transcription factor, only nuclear expression of TTF-1 was scored. Scoring was performed by a single pathologist (PSS) blinded to diagnosis and was recorded as a percentage of endometrial glandular cells with any nuclear expression of TTF-1. A positive case was defined as any nuclear TTF-1 expression in endometrial glandular cells present in each spot.

\section{Clinical Characteristics}

Clinically relevant information was extracted by medical record review and included smoking history, hypertension, diabetes, obesity, subfertility, cancer family history, gravidity, parity, body mass index, age at diagnosis, age of menopause, and age of menarche.

\section{Statistical Methods}

For statistical methods, StatView Version 5.0 (SAS Institute, Cary, NC, USA) and the statistical software R (http://www/r-project.org) were used. Nuclear positivity for each case was expressed as a percentage and was calculated from the mean pooled value of TTF-1 expression from which there were multiple informative spots as previously described. ${ }^{18} \mathrm{~A}$ positive case was defined as any nuclear TTF-1 expression in endometrial glandular cells present. For all results, $P<0.05$ was considered significant. MannWhitney $U$-test was used to compare differential 

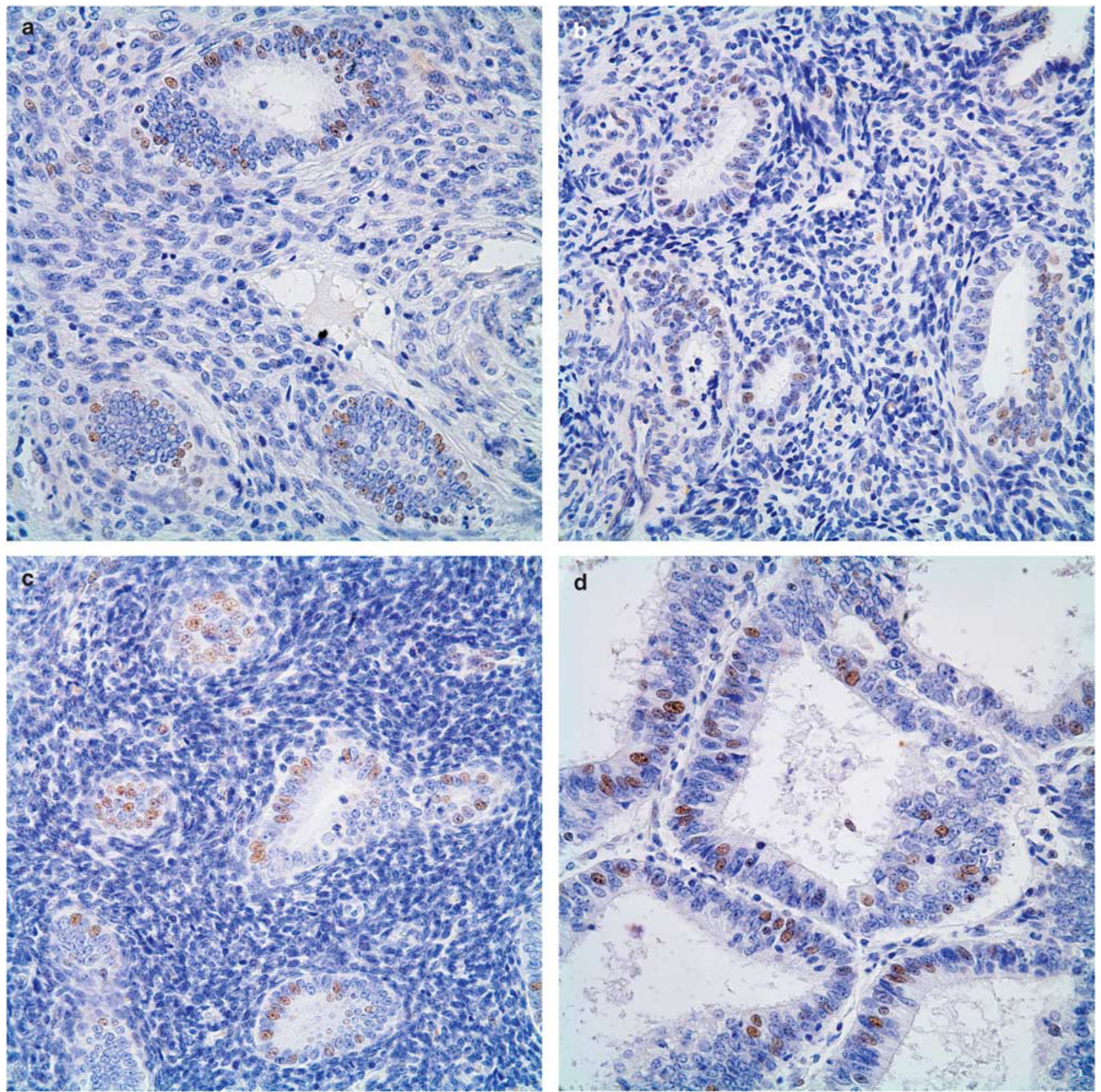

Figure 1 (a-f) TTF-1 expression in endometrium, representative cases. (a) Benign endometrium; (b) simple hyperplasia; (c) simple atypical hyperplasia; (d) complex hyperplasia; (e) complex atypical hyperplasia; (f) endometrial carcinoma. Immunohistochemical staining, $\times 400$.

expression of TTF-1 expression between endometrial lesions (eg, complex atypical hyperplasia vs adenocarcinoma). Log-rank test was applied to determine $P$-values from Kaplan-Meier plots for progression to cancer. Fisher's exact test was used to compare categorical variables (eg, dichotomized TTF-1 expression vs obesity). Univariate and multivariate Cox models were utilized for the comparison of categorical (eg, progression vs no progression to cancer) with continuous variables (eg, TTF-1 expression as a percentage).

For analysis of cancer progression, patients were excluded if they had no follow-up tissue after the initial sample collection and if their only follow-up time point was $<1$ year after the initial sample acquisition in order to exclude a false negative initial biopsy.

\section{Results}

\section{TTF-1 Expression in Benign, Hyperplastic, and Malignant Endometrium}

We examined the expression of TTF-1 in endometrial specimens that were derived from serial interval endometrial biopsies. Overall, 392 cases 


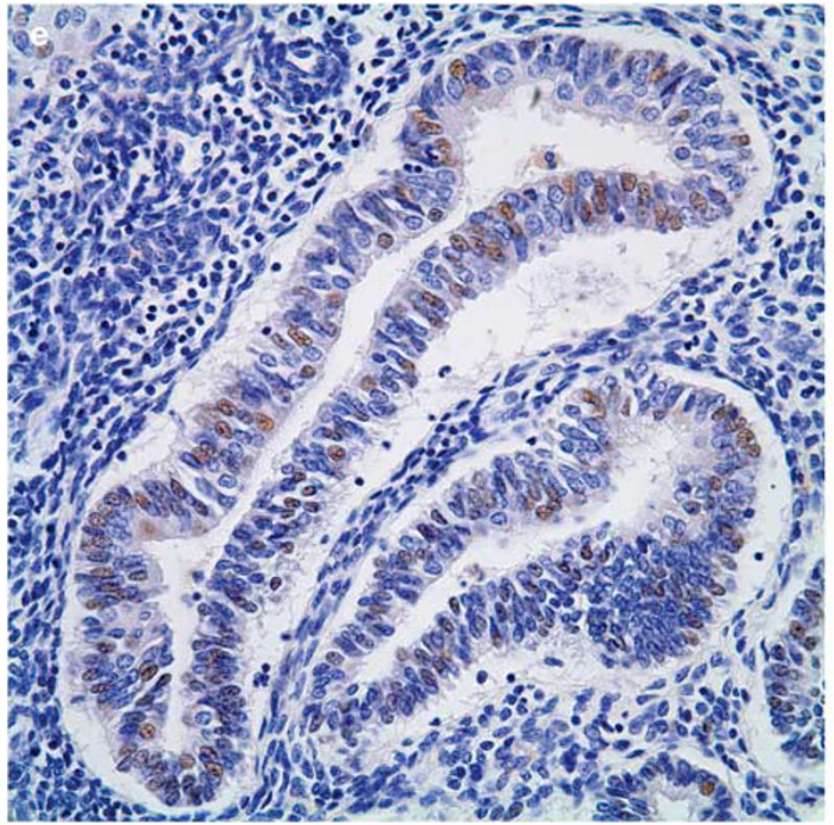

Figure 1 Continued.

had informative TTF-1 expression, including 216 benign cases, 45 hyperplasia cases, 25 atypical hyperplasia cases, and 106 endometrial carcinomas cases. We initially analyzed the percentage of specimens in each subgroup that had any nuclear expression of TTF-1. Based on this analysis, TTF-1 expression was detected in $55 \%$ of benign cases, $53 \%$ of hyperplasia cases, $52 \%$ of atypical hyperplasia cases, and $64 \%$ of endometrial carcinoma cases. Figure 1 shows examples of positive TTF-1 staining for the respective histopathological subgroups.

We next examined the percentage of cells that stained positive for TTF-1. Interestingly, the mean nuclear positivity increased with worsening endometrial abnormality from benign to hyperplasia, atypical hyperplasia, and carcinoma (Figure 2). Significant differences in nuclear positivity were found between benign endometrium and carcinoma $(P=0.0007)$, and non-atypical hyperplasia and carcinoma $(P=0.05)$. No significant differences were found between atypical hyperplasia and carcinoma $(P=0.68)$, benign and atypical hyperplasia $(P=0.35)$, benign and hyperplasia without atypia $(P=0.67)$, and hyperplasia with and without atypia $(P=0.54)$. Among the 81 patients with endometrial carcinoma, 56 were stage I, 6 were stage II, and 10 patients were stages III-IV. No correlation was found between TTF-1 expression and stage.

\section{TTF-1 Expression Is Correlated with Decreased Risk of Progression to Cancer}

We next examined whether expression of TTF-1 in any non-cancer histological subtype is correlated

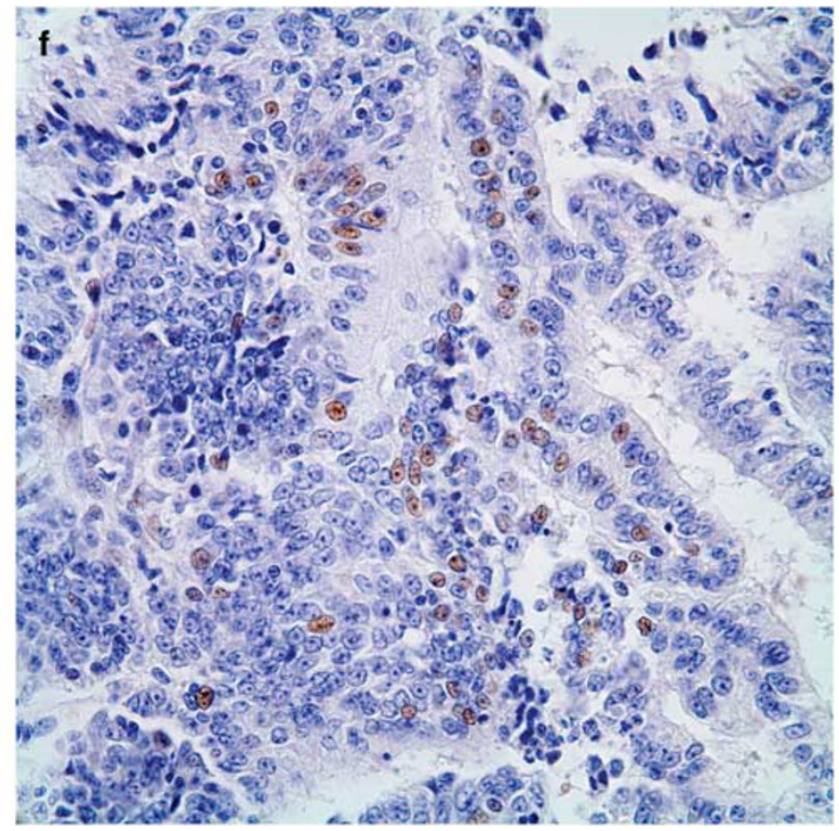

with risk of progression to cancer. Patients with at least one additional case and $>1$ year of follow-up were included in the analysis for progression to cancer. Surprisingly, when we considered TTF-1 expression in 108 cases of benign endometrium, the presence of TTF-1 expression was correlated with a decreased progression to cancer $(P=0.001$; Figure 3). In contrast, neither TTF-1 expression in non-atypical hyperplasia (simple hyperplasia, $n=26$ and complex hyperplasia, $n=14$ ) nor atypical hyperplasia (simple atypical hyperplasia and complex atypical hyperplasia, $n=23$ ) showed a correlation with progression to cancer.

\section{TTF-1 Expression and Association with Clinical Characteristics}

We next examined the relationship between TTF-1 expression and progression to cancer in the context of various clinical characteristics that are known to modulate the risk of developing endometrial carcinoma. We correlated TTF-1 expression in benign, hyperplastic, and malignant cases with smoking history, presence of hypertension, diabetes mellitus, obesity, subfertility, cancer family history, gravidity, parity, body mass index, age of menarche, and age at diagnosis. We found that later age of menopause was significantly correlated with TTF-1 expression in benign, hyperplastic, and malignant endometrium. Specifically, benign endometrial specimens from patients with mean age of menopause of $<50$ years were positive for TTF-1 in 30 of $42(71 \%)$ cases, while specimens from patients with a mean age of menopause $\geq 50$ years were positive for TTF- 1 in 30 of $58(52 \%)$ cases $(P=0.03)$. Similarly, hyperplasia 


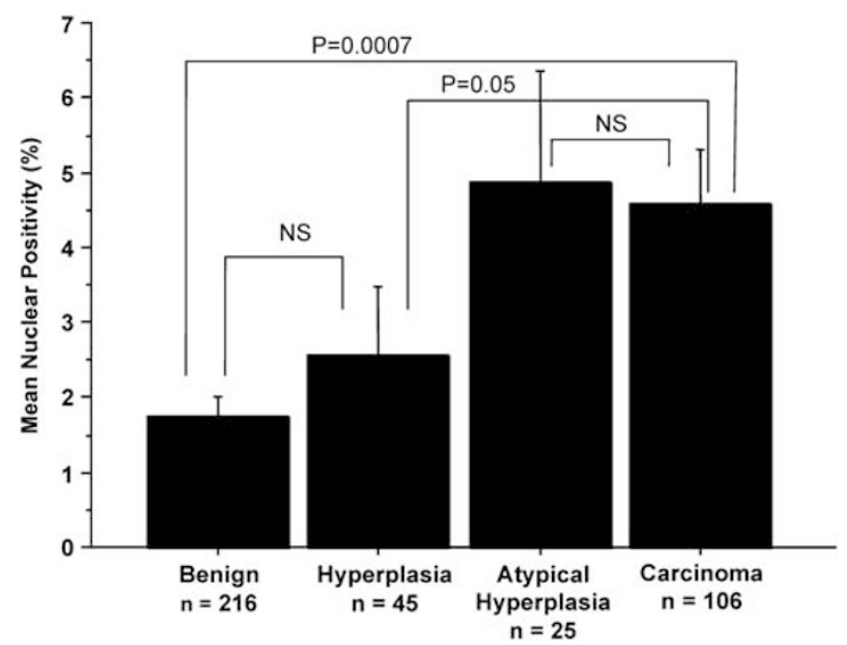

Figure 2 Mean nuclear positivity of TTF-1 expression in benign, hyperplastic, and malignant endometrium. Nuclear positivity is expressed as a percentage of endometrial glandular cell nuclei staining for TTF-1 for each case. The mean nuclear positivity is determined for each diagnostic category. $P$-values are indicated for comparison of two diagnostic categories as indicated by bracket lines. Error bars ( \pm 1 s.d.) are depicted for each mean. NS, not statistically significant.

specimens from patients with a mean age of menopause of $<50$ years showed a greater percentage of TTF-1-positive staining (17 of $22 \quad(77 \%)$ cases compared with 15 of 26 (58\%) cases, respectively; $P=0.04)$. Conversely, in carcinoma specimens, the percentage of TTF-1 positivity was greater in patients with a mean age of menopause $\geq 50$ years of age ( 27 of 34 cases, $79 \%$ ) compared to patients with mean age of menopause beginning $<50$ years of age (27 of 43 cases, $62.8 \% ; P=0.05$ ). In carcinoma specimens alone, body mass index and age of menarche were also found to correlate significantly with TTF-1 expression. Specifically, carcinoma specimens from patients with a mean body mass index $<30 \mathrm{~kg} / \mathrm{m}^{2}$ were positive for TTF-1 in 35 of 45 (78\%) cases, while specimens from those with a mean body mass index $\geq 30 \mathrm{~kg} / \mathrm{m}^{2}$ were positive for TTF-1 in 15 of 27 (56\%) cases $(P=0.02)$. In regards to age of menarche, patients with specimens that were positive for TTF-1 expression had a later mean age of menarche compared to patients with specimens that were negative for TTF-1 $(P=0.01)$. Since presence of TTF-1 was correlated with a decreased progression to cancer, we examined TTF-1 expression in the context of smoking history, a clinical characteristic known to be protective of cancer, and found that smoking history was positively correlated with the presence of TTF-1 expression in hyperplastic endometrium $(P=0.04)$, but not in benign or carcinoma specimens. Other factors listed in Table 1 did not show any correlation with TTF-1 expression.

We used a univariate analysis to examine whether TTF-1 expression or any clinical characteristics may be predictive of progression to cancer in our cohort (Table 2). We found a significant association

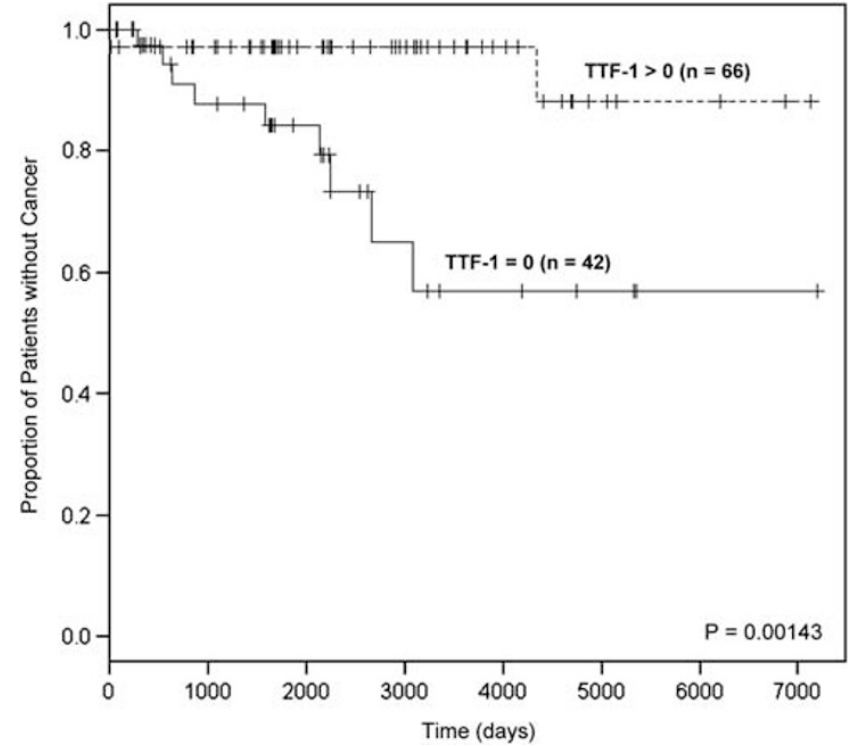

Figure 3 TTF-1 expression in benign endometrium and development of cancer. Kaplan-Meier curves show the percentage of patients with initially normal endometrium that remain without endometrial carcinoma. The hatched line represents patients with any nuclear TTF-1 expression in endometrial glandular cells. The solid line represents patients with no TTF-1 expression in endometrial glandular cells. Analysis was performed using a log-rank test; the $P$-value is indicated as shown.

between positive TTF-1 expression and decreased risk for progression to cancer (hazards ratio $(\mathrm{HR})=0.155$, CI: $0.042-0.577, P=0.001$ ). Later age at menopause was associated with a slightly decreased risk for progression to cancer (HR $=0.959$, CI: 0.920-0.999, $P=0.04$ ). Other clinical characteristics including smoking history, hypertension, diabetes mellitus, obesity, subfertility, cancer family history, gravidity, parity, body mass index, and age of menarche did not demonstrate an association with cancer progression.

We performed a multivariate analysis for the factors shown to be significantly associated with progression to cancer. We determined whether TTF-1 expression or age of menopause could independently predict cancer progression. We found that the absence of TTF-1 expression in benign cases was an independent predictor of cancer progression $(\mathrm{HR}=0.104,95 \%$ CI: 0.024-0.455, $P=0.003$ ) (Table 3). Later age of menopause was likewise identified as an independent predictive factor $(\mathrm{HR}=0.868$, CI: $0.778-0.968, P=0.01070$ ).

\section{Discussion}

Our study is the first to provide evidence that TTF-1 might elicit tumor suppressor function in normal endometrial tissue. We found that positive TTF-1 expression in benign endometrium is associated with decreased risk of endometrial cancer development. These findings suggest that TTF- 1 might affect 
Table 1 TTF-1 expression and correlation with clinical characteristics

\begin{tabular}{|c|c|c|c|c|c|c|c|c|c|}
\hline \multirow[b]{2}{*}{ TTF-1 result } & \multicolumn{3}{|c|}{ Benign } & \multicolumn{3}{|c|}{ Hyperplasia } & \multicolumn{3}{|c|}{ Carcinoma } \\
\hline & + & - & P-value & + & - & P-value & + & - & $\mathrm{P}$-value \\
\hline \multicolumn{10}{|l|}{ Smoking history } \\
\hline- & 8 & 22 & $0.12^{\mathrm{a}}$ & 26 & 10 & $0.04^{\mathrm{a}}$ & 32 & 13 & $0.62^{\mathrm{a}}$ \\
\hline+ & 16 & 16 & & 7 & 10 & & 19 & 10 & \\
\hline \multicolumn{10}{|l|}{ Hypertension } \\
\hline- & 37 & 22 & $0.84^{\mathrm{a}}$ & 13 & 13 & $0.16^{\mathrm{a}}$ & 30 & 14 & $>0.99^{\mathrm{a}}$ \\
\hline+ & 24 & 16 & & 20 & 8 & & 23 & 10 & \\
\hline \multicolumn{10}{|l|}{ Diabetes mellitus } \\
\hline- & 52 & 28 & $0.19^{\mathrm{a}}$ & 23 & 16 & $0.76^{\mathrm{a}}$ & 39 & 15 & $0.27^{\mathrm{a}}$ \\
\hline+ & 9 & 10 & & 10 & 5 & & 12 & 9 & \\
\hline \multicolumn{10}{|l|}{ Obesity } \\
\hline- & 34 & 24 & $>0.99^{\mathrm{a}}$ & 17 & 11 & $>0.99^{\mathrm{a}}$ & 35 & 10 & $0.12^{\mathrm{a}}$ \\
\hline+ & 26 & 17 & & 16 & 9 & & 18 & 12 & \\
\hline \multicolumn{10}{|l|}{ Subfertility } \\
\hline- & 28 & 22 & $>0.99^{\mathrm{a}}$ & 17 & 11 & $0.53^{\mathrm{a}}$ & 28 & 14 & $0.79^{\mathrm{a}}$ \\
\hline+ & 19 & 15 & & 14 & 5 & & 17 & 7 & \\
\hline \multicolumn{10}{|c|}{ Cancer family history } \\
\hline- & 31 & 22 & $0.53^{\mathrm{a}}$ & 17 & 11 & $0.77^{\mathrm{a}}$ & 32 & 13 & $>0.99^{\mathrm{a}}$ \\
\hline+ & 19 & 15 & & 16 & 8 & & 20 & 9 & \\
\hline \multicolumn{10}{|l|}{ Gravidity } \\
\hline Median (range) & $2(0-7)$ & $2(0-7)$ & $0.54^{\mathrm{b}}$ & $1(0-4)$ & $2(0-4)$ & $0.26^{\mathrm{b}}$ & $2(0-7)$ & $1.5(0-7)$ & $0.37^{\mathrm{b}}$ \\
\hline Mean & 2.4 & 2.0 & & 1.4 & 1.9 & & 2.2 & 2.0 & \\
\hline $0-1$ & 24 & 18 & & 21 & 7 & & 19 & 11 & \\
\hline$\geqslant 2$ & 35 & 23 & & 13 & 13 & & 35 & 11 & \\
\hline \multicolumn{10}{|l|}{ Parity } \\
\hline Median (range) & $1.5(0-5)$ & $1(0-4)$ & $0.97^{\mathrm{b}}$ & $0.5(0-4)$ & $2(0-3)$ & $0.09^{\mathrm{b}}$ & $1.5(0-4)$ & $1(0-5)$ & $0.72^{\mathrm{b}}$ \\
\hline Mean & 1.5 & 1.4 & & 1.0 & 1.6 & & 1.4 & 1.6 & \\
\hline $0-1$ & 30 & 22 & & 22 & 8 & & 27 & 12 & \\
\hline$\geqslant 2$ & 30 & 19 & & 12 & 12 & & 27 & 10 & \\
\hline \multicolumn{10}{|c|}{ Body mass index $\left(\mathrm{kg} / \mathrm{m}^{2}\right)$} \\
\hline Median (range) & $27(16-57)$ & $28(19-55)$ & $0.71^{\mathrm{b}}$ & $28(19-56)$ & $29(19-53)$ & $0.52^{\mathrm{b}}$ & $24.5(19-63)$ & $31.5(19-56)$ & $0.02^{b}$ \\
\hline Mean & 28.5 & 29.5 & & 29.9 & 32.1 & & 28.5 & 33.3 & \\
\hline $\mathrm{BMI}<30$ & 34 & 25 & & 17 & 11 & & 35 & 10 & \\
\hline $\mathrm{BMI} \geqslant 30$ & 25 & 15 & & 15 & 9 & & 15 & 12 & \\
\hline \multicolumn{10}{|c|}{ Diagnosis age (years) } \\
\hline Median (range) & $47(30-56)$ & $47(30-75)$ & $0.35^{\mathrm{b}}$ & $48(35-74)$ & $51(34-71)$ & $0.10^{\mathrm{b}}$ & $53(38-85)$ & $47.5(30-74)$ & $0.11^{\mathrm{b}}$ \\
\hline Mean & 46.27 & 48.09 & & 47.6 & 50.8 & & 53.3 & 49.0 & \\
\hline \multicolumn{10}{|c|}{ Menopause age (vears) } \\
\hline Median (range) & $50(31-55)$ & $51(33-57)$ & $\mathbf{0 . 0 3}^{\mathrm{b}}$ & $49(31-53)$ & $51(36-59)$ & $\mathbf{0 . 0 4}^{\mathrm{b}}$ & $49.5(40-63)$ & $48(33-55)$ & $0.05^{b}$ \\
\hline Mean & 48.5 & 50.2 & & 47.6 & 50.6 & & 49.2 & 45.9 & \\
\hline$<50$ & 30 & 12 & & 17 & 5 & & 27 & 16 & \\
\hline$\geqslant 50$ & 30 & 28 & & 15 & 11 & & 27 & 7 & \\
\hline \multicolumn{10}{|c|}{ Menarche age (vears) } \\
\hline Median (range) & $12(9-15)$ & $12(8-16)$ & $0.75^{\mathrm{b}}$ & $12(10-15)$ & $12(11-16)$ & $0.62^{\mathrm{b}}$ & $12(10-16)$ & $12(8-14)$ & $0.01^{b}$ \\
\hline Mean & 12.1 & 12.2 & & 12.2 & 12.2 & & 12.5 & 11.7 & \\
\hline
\end{tabular}

Unless indicated, numbers represent number of patients with a particular clinical characteristic. Bolded values are significant $P$-values. + , positive; -, negative. ${ }^{\mathrm{a} F i s h e r ' s ~ e x a c t ~ t e s t . ~}{ }^{\mathrm{b}} \mathrm{Mann}-$ Whitney test.

the process of malignant transformation in normal endometrial cells.

Other investigators have reported positive TTF-1 expression in gynecologic tissues and tumors. In a review of the literature, early studies examining the utility of TTF-1 expression as a diagnostic aid for metastatic lung adenocarcinoma and small cell carcinoma noted positive TTF-1 nuclear expression 
Table 2 Univariate Cox models for clinical variables

\begin{tabular}{llcc}
\hline & \multicolumn{3}{c}{ Benign endometrium $(\mathrm{n}=109)$} \\
\cline { 2 - 4 } & P-value & Hazard ratio & $95 \%$ CI \\
\hline Smoking history & 0.6124 & 1.130 & $0.703-1.818$ \\
Hypertension & 0.6306 & 1.117 & $0.712-1.752$ \\
Diabetes & 0.2327 & 1.364 & $0.817-2.277$ \\
Obesity & 0.8579 & 0.959 & $0.608-1.514$ \\
Subfertility & 0.2011 & 0.714 & $0.425-1.199$ \\
Cancer family history & 0.5369 & 0.864 & $0.544-1.374$ \\
Breast cancer & 0.9301 & 1.032 & $0.515-2.066$ \\
Colon cancer & 0.4808 & 1.650 & $0.404-6.757$ \\
Gravidity $^{\mathrm{a}}$ & 0.2864 & 0.931 & $0.817-1.062$ \\
Parity $^{\mathrm{a}}$ & 0.4710 & 0.940 & $0.794-1.112$ \\
Body mass index $^{\mathrm{a}}$ & 0.2652 & 1.015 & $0.989-1.042$ \\
Age of menopause $^{\mathrm{a}}$ & $\mathbf{0 . 0 4 4 4}$ & 0.959 & $0.920-0.999$ \\
Age of menarche $^{\mathrm{a}}$ & 0.5241 & 1.060 & $0.886-1.267$ \\
TTF-1 positive & $\mathbf{0 . 0 0 1 4}$ & 0.155 & $0.042-0.577$ \\
\hline
\end{tabular}

CI, confidence interval.

${ }^{\mathrm{a}}$ All these variables were analyzed as continuous variables. Bolded values are significant $P$-values.

Table 3 Multivariate Cox model for clinical variables

\begin{tabular}{lccc}
\hline & \multicolumn{3}{c}{ Benign endometrium $(\mathrm{n}=109)$} \\
\cline { 2 - 4 } & P-value & Hazard ratio & $95 \%$ CI \\
\hline Age of menopause & $\mathbf{0 . 0 1 0 7}$ & 0.868 & $0.778-0.968$ \\
TTF-1 positive & $\mathbf{0 . 0 0 2 7}$ & 0.104 & $0.024-0.455$
\end{tabular}

CI, confidence interval.

Bolded values are significant $P$-values.

in a small number of gynecologic cancer cases. ${ }^{20-22}$ More recent studies have reported positive TTF-1 expression ranging from $0 \%{ }^{12}$ to $22 \%$ in endometrial endometrioid adenocarcinomas, ${ }^{10,11,14,23}$ $3 \%{ }^{12}$ to $23 \%$ in endometrial serous carcinomas, ${ }^{11,14}$ $0 \%{ }^{12}$ to $7 \%$ in endocervical adenocarcinomas, ${ }^{10,14,23}$ $82 \%$ in endometrial malignant mixed müllerian tumors, ${ }^{11}$ and $0 \%$ in uterine leiomyosarcomas. ${ }^{10} \mathrm{In}$ ovarian neoplasms, positive TTF-1 expression was reported in serous, endometrioid, clear cell, and mucinous adenocarcinomas (ranging from $1 \%{ }^{24}$ to $13 \%^{10,11,13}$ of all histologic subtypes) and in mucinous cystadenomas, ${ }^{11}$ while negative TTF-1 expression was observed in mucinous tumors of low malignant potential ${ }^{13}$ and serous cystadenomas. ${ }^{11}$ Other investigators have shown that the percentage of cases with positive TTF-1 expression varies depending on the antibody clone, detection system, and specimen type (tissue microarray vs whole tissue section) utilized. ${ }^{11,13}$

Zhang et $a l^{11}$ first reported positive TTF-1 expression in non-neoplastic gynecologic tissues (two benign fallopian tubes and one benign polypoid endometrium) and in a later study examined TTF-1 expression in 113 non-neoplastic gynecologic tissues in patients without a history or family history of gynecologic or breast cancer. In their study, 96\% of fallopian tube tissues and $43 \%$ of endocervical tissues had positive TTF-1 expression. In addition, $75 \%$ of proliferative endometrium and $55 \%$ of secretory endometrium cases were positive for TTF-1 nuclear expression while all inactive endometrium cases were negative for TTF- 1 expression. ${ }^{15}$

Although others have described TTF-1 expression in gynecologic tissues and tumors, the biological function of TTF-1 in normal endometrial tissue is unclear. Interestingly, studies of other cancer diseases have reported that the expression of TTF-1 confers a clinically less aggressive phenotype and a more favorable prognosis. In thyroid carcinomas for example, TTF-1 was shown to be present in the vast majority of well-differentiated papillary and follicular carcinomas while it was generally absent in undifferentiated anaplastic thyroid carcinomas. ${ }^{7}$ In lung adenocarcinomas, increased TTF-1 expression was found to be associated with a better prognosis, particularly in early stages. ${ }^{25-27}$ Finally, Fujiwara et $a l^{10}$ demonstrated in a univariate analysis that the presence of TTF-1 in ovarian epithelial malignancies was associated with a significantly better overall and progression-free survival. These studies support the hypothesis that TTF-1 expression reduces the aggressive malignant behavior of cancer cells.

The biological mechanisms underlying the tumor suppressor function of TTF-1 remain to be elucidated, but recent studies in preclinical animal models have provided further insights. Saito et $a l,{ }^{16}$ for example, demonstrated that TTF-1 inhibited the TGF- $\beta$-mediated epithelial-mesenchymal transition, and restored an epithelial phenotype in lung adenocarcinomas, supporting a protective role for TTF-1 in cancer progression. Furthermore, Winslow et $a l^{17}$ demonstrated in a murine lung adenocarcinoma model that TTF-1 downregulation was linked to increased de-differentiation and metastatic potential.

Endometrioid-type endometrial adenocarcinomas are usually estrogen dependent, and prolonged, unopposed exposure to estrogens is one of the established risk factors. We found a positive association between TTF-1 expression and clinical conditions that lead to lower, cumulative estrogen exposure, including earlier onset of menopause, later menarche, and a lower body mass index. These observations suggest that estrogens might play a role in the regulation of TTF-1 expression. Our finding that hyperplastic lesions with positive TTF-1 expression are associated with patients who have a positive smoking history is interesting in this context since smoking reduces the risk of endometrial cancer development. The protective effect of smoking has been attributed to decreased bioavailability of estrogenic compounds in endometrial tissue; ${ }^{28}$ however, further studies will be necessary to show whether smoking could potentially upregulate TTF-1 expression in hyperplastic endometrium and whether a low estrogen endometrial microenvironment would mediate this effect. Interestingly, estrogen hormone replacement therapy has been 
shown to be associated with a shorter survival in lung cancer patients. ${ }^{29}$

The identification of biological markers in normal and hyperplastic endometrium that reliably predict an increased risk of progression to endometrial cancer would provide important clinical benefits. The tumor suppressor gene PTEN has been extensively studied in endometrial cancer. ${ }^{30-33}$ Early studies by Mutter et al ${ }^{34}$ demonstrated gradual loss of quantitative PTEN protein expression during progression from benign endometrium to hyperplasia and endometrial cancer. However, it is unclear whether loss of PTEN protein expression or the presence of PTEN inactivating mutations in endometrial cancer precursor lesions is predictive of progression. ${ }^{32}$ A recent case-controlled study demonstrated PTEN loss of expression may not be associated with progression. $^{35}$ Other studies have linked PTEN expression with clinical characteristics known to be protective of endometrial carcinoma. ${ }^{33}$

In summary, we have found the expression of TTF-1 in benign endometrium is an independent predictor of progression to endometrial carcinoma. While the data are preliminary and should be validated in another well-annotated cohort, our findings suggest that TTF-1 might function as a tumor suppressor protein in endometrial cancer. Furthermore, TTF-1 might provide useful clinical information as a predictive marker in endometrial cancer progression. Future studies are needed to further evaluate the biological function of TTF-1 in endometrial tissue and prospectively assess its potential role as a prognostic marker.

\section{Acknowledgements}

We thank Sarah Dry and the UCLA Translational Pathology Core Laboratory for facilitating the initial efforts of this study and for technical assistance with immunohistochemistry. We also thank Wafic El Masri, Amer Karam, and Michael Fishbein for providing invaluable comments during manuscript preparation.

\section{Disclosure/conflict of interest}

The authors declare no conflict of interest.

\section{References}

1 Siegel R, Ward E, Brawley O, et al. Cancer statistics, 2011: the impact of eliminating socioeconomic and racial disparities on premature cancer deaths. CA Cancer J Clin 2011;61:212-236.

2 Ronnett BM, Zaino RJ, Ellenson LH, et al. Endometrial carcinoma. In: Kurman RJ, Ellenson LH, Ronnett BM (eds). Blaustein's Pathology of the Female Genital Tract 6th edn. Springer: New York, 2011, pp 395-396.
3 Zaino RJ, Kauderer J, Trimble CL, et al. Reproducibility of the diagnosis of atypical endometrial hyperplasia: a Gynecologic Oncology Group study. Cancer 2006; 106:804-811.

4 Allison KH, Reed SD, Voigt LF, et al. Diagnosing endometrial hyperplasia: why is it so difficult to agree? Am J Surg Pathol 2008;32:691-698.

5 Sherman ME, Ronnett BM, Ioffe OB, et al. Reproducibility of biopsy diagnoses of endometrial hyperplasia: evidence supporting a simplified classification. Int J Gynecol Pathol 2008;27:318-325.

6 Boggaram V. Thyroid transcription factor-1 (TTF-1/ Nkx2.1/TITF1) gene regulation in the lung. Clin Sci 2009;116:27-35.

7 Ordonez NG. Thyroid transcription factor-1 is a marker of lung and thyroid carcinoma. Adv Anat Pathol 2000; 7:123-127.

8 Agoff SN, Lamps LW, Philip AT, et al. Thyroid transcription factor-1 is expressed in extrapulmonary small cell carcinomas but not in other extrapulmonary neuroendocrine tumors. Mod Pathol 2000;13:238-242.

9 Robens J, Goldstein L, Gown AM, et al. Thyroid transcription factor-1 expression in breast carcinomas. Am J Surg Pathol 2010;34:1881-1885.

10 Fujiwara S, Nawa A, Nakanishi T, et al. Thyroid transcription factor 1 expression in ovarian carcinomas is an independent prognostic factor. Hum Pathol 2010; 41:560-565.

11 Zhang PJ, Gao HG, Pasha TL, et al. TTF-1 expression in ovarian and uterine epithelial neoplasia and its potential significance, an immunohistochemical assessment with multiple monoclonal antibodies and different secondary detection systems. Int J Gynecol Pathol 2008;28:10-18.

12 Alkushi A, Irving J, Hsu F, et al. Immunoprofile of cervical and endometrial adenocarcinomas using a tissue microarray. Virchows Arch 2003;442:271-277.

13 Kubba LA, McCluggage WG, Liu J, et al. Thyroid transcription factor-1 expression in ovarian epithelial neoplasms. Mod Pathol 2008;21:485-490.

14 Siami K, McCluggage WG, Ordonez NG, et al. Thyroid transcription factor-1 expression in endometrial and endocervical adenocarcinomas. Am J Surg Pathol 2007;31:1759-1763.

15 Niu H, Pasha TL, Pawel BR, et al. Thyroid transcription factor-1 expression in normal and gynecologic tissues and its potential significance. Int J Gynecol Pathol 2009;28:301-307.

16 Saito RA, Watabe T, Horiguchi K, et al. Thyroid transcription factor-1 inhibits transforming growth factor-beta-mediated epithelial-to-mesenchymal transition in lung adenocarcinoma cells. Cancer Res 2009;69:2783-2791.

17 Winslow MM, Dayton TL, Verhaak RGW, et al. Suppression of lung adenocarcinoma progression by Nkx2-1. Nature 2011;473:101-108.

18 Habeeb O, Goodglick L, Soslow RA, et al. Epithelial membrane protein-2 expression is an early predictor of endometrial cancer development. Cancer 2010;116: 4718-4726.

19 Silverberg SG, Kurman RJ, Nogales F, et al. Tumors of the uterine corpus. In: Tavassoli FA, Devilee P (eds). World Health Organization Classification of Tumors, Pathology and Genetics, Tumours of the Breast and Female Genital Organs. IARC Press: Lyon, France, 2003. 20 Bejarano PA, Baughman RP, Biddinger PW, et al. Surfactant proteins and thyroid transcription factor-1 
in pulmonary and breast carcinomas. Mod Pathol 1996;9:445-452.

21 Kaufmann O, Dietel M. Expression of thyroid transcription factor-1 in pulmonary and extrapulmonary small cell carcinomas and other neuroendocrine carcinomas of various primary sites. Histopathology 2000;36:415-420.

22 Hecht JL, Pinkus JL, Weinstein LJ, et al. The value of thyroid transcription factor-1 in cytologic preparations as a marker for metastatic adenocarcinoma of lung origin. Am J Clin Pathol 2001;116:483-488.

23 Han CP, Kok LF, Lee MY, et al. Five commonly used markers (p53, TTF1, CK7, CK20, and CK34betaE12) are of no use in distinguishing between primary endocervical and endometrial adenocarcinomas in a tissue microarray extension study. Arch Gynecol Obstet 2010;281:317-323.

24 Graham AD, Williams ARW, Salter DM. TTF-1 expression in primary ovarian epithelial neoplasia (correspondence). Histopathology 2006;48:764-776.

25 Berghmans T, Paesmans M, Mascaux C, et al. Thyroid transcription factor 1-a new prognostic factor in lung cancer: a meta-analysis. Ann Oncol 2006;17: 1673-1676.

26 Perner S, Wagner PL, Soltermann A, et al. TTF1 expression in non-small cell lung carcinoma: association with TTF1 gene amplification and improved survival. J Pathol 2009;217:65-72.

27 Anagnostou VK, Syrigos KN, Bepler G, et al. Thyroid transcription factor 1 is an independent prognostic factor for patients with stage I lung adenocarcinoma. J Clin Oncol 2009;27:271-278.
28 Baron JA, La Vecchia C, Levi F. The antiestrogenic effect of cigarette smoking in women. Am J Obstet Gynecol 1990;162:502-514.

29 Ganti AK, Sahmoun AE, Panwalkar AW, et al. Hormone replacement therapy is associated with decreased survival in women with lung cancer. J Clin Oncol 2006;24:59-63.

30 Minaguchi T, Nakagawa S, Takazawa Y, et al. Combined phospho-Akt and PTEN expressions associated with post-treatment hysterectomy after conservative progestin therapy in complex atypical hyperplasia and stage Ia, G1 adenocarcinoma of the endometrium. Cancer Lett 2007;248:112-122.

31 Monte NM, Webster KA, Neuberg D, et al. Joint loss of PAX2 and PTEN expression in endometrial precancers and cancer. Cancer Res 2010;70:6225-6232.

32 Llobet D, Pallares J, Yeramian A, et al. Molecular pathology of endometrial carcinoma: practical aspects from the diagnostic and therapeutic viewpoints. J Clin Pathol 2009;62:777-785.

33 Dellas A, Jundt G, Sartorius G, et al. Combined PTEN and p27kip1 protein expression patterns are associated with obesity and prognosis in endometrial carcinomas. Clin Cancer Res 2009;15:2456-2462.

34 Mutter GL, Lin MC, Fitzgerald JT, et al. Altered PTEN expression as a diagnostic marker for the earliest endometrial precancers. J Natl Cancer Inst 2000;92: 924-931.

35 Lacey Jr JV, Mutter GL, Ronnett BM, et al. PTEN expression in endometrial biopsies as a marker of progression to endometrial carcinoma. Cancer Res 2008;68: 6014-6020. 